\title{
Open hybrid inflation
}

\author{
Juan García-Bellido \\ Theory Division, CERN, CH-1211 Geneva 23, Switzerland
}

Andrei Linde

Department of Physics, Stanford University, Stanford, California 94305-4060

(Received 23 January 1997)

\begin{abstract}
We propose an open hybrid inflation scenario that produces an open universe with a "tilted" $n>1$ spectrum of metric perturbations. The model contains a symmetry-breaking field that tunnels to its true vacuum, producing a single bubble inside which hybrid inflation drives the universe to almost flatness. In order to obtain density perturbations with $n>1$ we analyze a recently proposed new version of hybrid inflation scenario called tilted hybrid inflation. In this scenario, unlike in the previously known versions of hybrid inflation, a considerable tilt of the spectrum can be obtained without fine tuning. The stage of inflation in this model is rather short, which allows us to obtain an inflationary universe with $\Omega<1$ in a more natural way. We study the separate contribution of scalar perturbations coming from the continuum subcurvature modes, the discrete supercurvature mode, and the bubble wall mode to the angular power spectrum of temperature fluctuations in open inflation. We derive bounds on the parameters of the model so that the predicted spectrum is compatible with the observed anisotropy of the microwave background. [S0556-2821(97)04812-1]
\end{abstract}

PACS number(s): $98.80 . \mathrm{Cq}$

\section{INTRODUCTION}

Until recently, inflation was associated unequivocally with a flat universe, due to its tendency to drive the spatial curvature so effectively to zero. However, it is now understood that inflation comprises a wider class of models, some of which may give rise to an open universe at present [1-3]. Such models generically contain a field trapped in a false vacuum, which tunnels to its true vacuum via the nucleation of a single bubble inside which a second period of inflation drives the universe to almost flatness. These models recently became rather popular because in an open universe it is possible to reconcile a large value of the Hubble constant [4] with the large estimated age of globular clusters [5]. But even independently of this issue, open inflation models have several interesting features that single them out from other cosmological models.

For a long time it seemed impossible to make any physical sense of the (mathematically consistent) open universe model because it presumed that an infinite universe appeared from nowhere at a single moment of time, being perfectly synchronized over an infinitely large length scale. This leads to the horizon problem in its strongest form. Also, whereas it seemed possible that a small closed universe could be created by "tunneling from nothing" and then experience a period of inflation, a similar event for an infinite open universe seemed impossible. Indeed, one may argue that such processes should be suppressed by $e^{-|S|}$, where $S$ is the action of the instanton describing the universe creation [6]. Therefore one could expect that quantum creation of an infinitely large open universe should be forbidden because this would involve tunneling with infinite action. The models of Refs. [1-3] show for the first time that a consistent physical model of an infinite homogeneous universe is possible, and that such a universe can appear as a result of quantum tunneling. The bubble containing an open universe looks finite from the point of view of an outside observer, and the probability of its creation is finite. Meanwhile an observer inside the bubble would see an infinite open universe.

Another distinct feature of such models is that the homogeneity problem is solved not through the exponential expansion, as in the usual inflationary models, but through the exponential suppression of the probability of production of spherically asymmetric bubbles [3]. This way one solves the homogeneity problem independently from the flatness problem. The origin of structure is still related to amplified quantum fluctuations of the field that drives inflation inside the bubble $[7,2]$. However, in the spectrum of temperature fluctuations there appears a new set of discrete supercurvature modes [8,9], associated with the open de Sitter vacuum $[7,10]$ and the bubble wall fluctuations at tunneling $[3,11-$ 13], which could be made small in some of the models [14].

There is some evidence that the observations made in a wide range of scales, from horizon size to large clusters of galaxies, constrain open inflation models (with small $\left.\Omega_{0} \sim 0.3-0.4\right)$ to have a 'tilted' spectrum of density perturbations with the spectral index $n>1$ [15], and essentially no other contribution, either from gravitational waves or supercurvature modes. The open inflation models considered so far predict a tilted $n<1$ spectrum, see, e.g., Ref. [16], which may contradict observational data for models with very small $\Omega_{0}[15]$.

In this paper, we consider a model of open inflation in which the second stage inside the bubble is driven by hybrid inflation [17]. Models of hybrid inflation are known to provide a blue tilted spectrum of metric perturbations, with negligible contribution of gravitational waves [18-21]. However, in most of the hybrid inflation models developed so far the tilt is extremely small; in order to achieve a considerable tilt of the spectrum in hybrid inflation one needs to fine tune the parameters of the model [20]. In what follows we will consider a new class of hybrid inflation models where a sig- 
nificant tilt can be achieved in a natural way, which we call tilted hybrid inflation, see Ref. [22]. We will make tilted hybrid inflation a part of an open inflation scenario. To make sure that supercurvature and bubble wall modes do not distort the cosmic microwave background (CMB) too much, we compute the angular power spectrum of temperature anisotropies for both the continuum modes and the supercurvature modes and find that there are models in which all constraints are satisfied.

Apart from scalar metric perturbations, open inflation also produces a primordial spectrum of gravitational waves, whose amplitude and scale dependence in single-bubble open inflation models has only recently been known [23]. In order to compare with observations there still remains to be computed the corresponding angular power spectrum of CMB temperature fluctuations. We will consider the constraints on the parameters of tilted hybrid models from such a tensor component of the CMB anisotropies in a future publication.

\section{TILTED HYBRID INFLATION}

The simplest realization of hybrid inflation in the context of the chaotic inflation scenario is provided by the potential [17]

$$
V(\phi, \psi)=\frac{1}{4 \lambda}\left(M^{2}-\lambda \psi^{2}\right)^{2}+\frac{1}{2} m^{2} \phi^{2}+\frac{1}{2} g^{2} \phi^{2} \psi^{2} .
$$

The bare masses $m$ and $M$ of the scalar fields $\phi$ and $\psi$ are "dressed" by their mutual interaction. At large values of the fields, their effective masses squared are both positive and the potential has the symmetry $\psi \leftrightarrow-\psi$. At small values of the field $\phi$, the potential has a maximum at $\phi=\psi=0$ and a global minimum at $\phi=0, \psi=\psi_{0} \equiv M / \sqrt{\lambda}$, where the above symmetry is broken.

Equations of motion for the homogeneous fields at the stage of their slow rolling during inflation are

$$
\begin{gathered}
3 H \dot{\phi}=-\left(m^{2}+g^{2} \psi^{2}\right) \phi, \\
3 H \dot{\psi}=\left(M^{2}-g^{2} \phi^{2}-\lambda \psi^{2}\right) \psi,
\end{gathered}
$$

where the Hubble constant is given by $H^{2}=8 \pi V / 3 M_{P}^{2}$. Motion starts at large $\phi$, where the effective mass squared of the $\psi$ field is positive and the field is sitting at the minimum of the potential at $\psi=0$. As the field $\phi$ decreases, its quantum fluctuations produce an almost scale invariant but slightly tilted spectrum of density perturbations [17-21].

During the slow roll of the field $\phi$, the effective mass of the triggering field is $m_{\psi}^{2}=g^{2} \phi^{2}-M^{2}$. When the field $\phi$ acquires the critical value $\phi_{c} \equiv M / g$, fluctuations of the massless $\psi$ field trigger the symmetry-breaking phase transition that ends inflation. If the bare mass $M$ of the $\sigma$ field is large compared with the rate of expansion $H$ of the universe, the transition will be instantaneous and inflation will end abruptly, as in the original hybrid inflation model [17]. If on the contrary the bare mass $M$ is of the order of $H$, then the transition will be very slow and there is the possibility of having yet a few more $e$ folds of inflation after the phase transition, see Refs. [24,25].
When $\psi=0$ the inflaton potential becomes $V(\phi)=M^{4} / 4 \lambda+m^{2} \phi^{2} / 2$. Since the scalar field $\phi$ takes relatively small values, for $m^{2} \ll g^{2} M^{2} / \lambda$ the energy density is dominated by the vacuum energy

$$
H_{0}^{2}=\frac{2 \pi M^{4}}{3 \lambda M_{P}^{2}},
$$

to very good accuracy [20]. It is then possible to integrate exactly the evolution equation of $\phi$ :

$$
\begin{gathered}
\phi(N)=\phi_{c} \exp (r N), \\
r=\frac{3}{2}-\sqrt{\frac{9}{4}-\frac{m^{2}}{H_{0}^{2}}} \simeq \frac{m^{2}}{3 H_{0}^{2}},
\end{gathered}
$$

where $N=H_{0}\left(t_{e}-t\right)$ is the number of $e$ folds to the end of inflation at $\phi=\phi_{c}$, and we have used the approximation $m \lesssim H_{0}$.

An important feature of the perturbation spectrum of the hybrid inflation model is its growth at large $k$; for example, at the end of inflation in this theory one has $[17,18]$

$$
\frac{\delta \rho}{\rho}(k)=\frac{2 \sqrt{6 \pi} g M^{5}}{5 \lambda \sqrt{\lambda} m^{2} M_{P}^{3}}\left(\frac{k}{H_{0}}\right)^{m^{2} / 3 H_{0}^{2}},
$$

which corresponds to a spectral index

$$
n \simeq 1+\frac{2 m^{2}}{3 H_{0}^{2}}=1+\frac{\lambda m^{2} M_{P}^{2}}{\pi M^{4}} .
$$

One should note that even though the spectral index $n$ in this model is greater than 1, for typical values of the parameters, $n=1$ with great accuracy. For example, one may consider the numerical values of parameters for the version of the hybrid inflation model discussed in Ref. [17]: $g^{2} \sim \lambda \sim 10^{-1}, m \sim 10^{2} \mathrm{GeV}$ (electroweak scale), $M \sim 10^{11}$ $\mathrm{GeV}$. In this case $n-1 \sim 10^{-4}$. Thus it is not very easy to use hybrid inflation in order to obtain a blue spectrum without fine tuning; in fact one could even argue that hybrid inflation has a very stable prediction that the spectrum must be almost exactly flat; see, however, Ref. [20].

This conclusion could be somewhat premature. Indeed, one can follow the lines of Ref. [26] and consider models where the field $\phi$ during inflation acquires an effective mass squared:

$$
m_{\mathrm{eff}}^{2}=m^{2}+\alpha H^{2}
$$

with $\alpha=O(1)$. This is a very natural assumption, which is true in a large class of supersymmetric models [27]. It is also true for models where the field $\phi$ has a nonminimal coupling with gravity described by the additional term $-\xi R \phi^{2} / 2$ in the Lagrangian. During hybrid inflation $R=12 H_{0}^{2}$, and the field $\phi$ acquires an effective mass (9) with $\alpha=12 \xi$. In such models one should consider those parts of the universe where $\phi \ll M_{P} / \sqrt{\alpha}$, because at much larger values of $\phi$ the effective gravitational constant $G^{-1}=M_{P}^{2} / 16 \pi-\xi \phi^{2} / 2$ becomes singular. For $\phi \ll M_{P} / \sqrt{\alpha}$ the Hubble constant can be approximated by Eq. (4). In such a case one has 


$$
\frac{\delta \rho}{\rho}(k)=\frac{9 \sqrt{2} H}{5 \pi \alpha \phi}=\frac{3 \sqrt{6} g M}{5 \sqrt{\lambda \pi} \alpha M_{P}}\left(\frac{k}{H_{0}}\right)^{\alpha / 3} .
$$

Here $\phi$ is the value of the scalar field at the moment when the perturbations are produced which at the end of inflation have momentum $k$. These perturbations have spectral index $n-1 \simeq 2 \alpha / 3>0$ for $\alpha \lesssim 1$. We call such models tilted hybrid inflation [22]: they provide a spectrum of density perturbations with a positive tilt, which may be large and may not require any fine tuning of parameters.

We will concentrate here, for definiteness, on a particular version of the tilted hybrid model proposed in Ref. [22]. This model has a potential for the field $\phi$ (while $\psi=0$, i.e., during the stage of inflation in our scenario), which is given by

$$
V(\phi)=V_{0} \exp \left(\frac{4 \pi \alpha \phi^{2}}{3 M_{P}^{2}}\right),
$$

where $V_{0}=M^{4} / 4 \lambda$ is the vacuum energy, and we are assuming $4 \pi \alpha \phi^{2} / 3 \ll M_{P}^{2}$. The effective mass of $\phi$ becomes

$$
m^{2}=V^{\prime \prime}(\phi)=\alpha H^{2}\left(1+\frac{8 \pi \alpha \phi^{2}}{3 M_{P}^{2}}\right) \simeq \alpha H^{2} .
$$

The field evolves according to Eq. (5) with $r \simeq \alpha / 3$, and $\dot{\phi}^{2}+m^{2} \phi^{2} \ll V_{0}$ is satisfied during inflation. In this model, the condition for inflation to occur $-\dot{H}<H^{2}$ is

$$
\phi<\phi_{\mathrm{inf}} \equiv \frac{3}{2 \sqrt{\pi} \alpha} M_{P}
$$

As a consequence, the number of $e$ folds has a maximum value

$$
N_{\max } \equiv \frac{3}{\alpha} \ln \left(\frac{\phi_{\mathrm{inf}}}{\phi_{c}}\right)=\frac{3}{\alpha} \ln \left(\frac{3 g M_{P}}{2 \sqrt{\pi} \alpha M}\right) .
$$

In this model, the spectrum of curvature perturbations for a flat universe is expressed by [22]

$$
\mathcal{P}_{\mathcal{R}}(k)=\frac{9 H^{2}(\phi)}{4 \pi^{2} \alpha^{2} \phi^{2}} .
$$

In a flat universe, the Cosmic Background Explorer (COBE) normalization imposes a constraint on the amplitude (15) (see Ref. [22]):

$$
\frac{3 g^{2} M^{2} e^{-2 \alpha N / 3}}{2 \lambda \alpha^{2} M_{P}^{2}} \simeq 10^{-8},
$$

where $N$ corresponds to the scale of the horizon today,

$$
N_{\text {hor }} \simeq 55+\frac{2}{3} \ln \left(\frac{V^{1 / 4}}{10^{16} \mathrm{GeV}}\right)+\frac{1}{3} \ln \left(\frac{T_{\mathrm{rh}}}{10^{9} \mathrm{GeV}}\right) .
$$

As we will see in Sec. IV, the constraint in an open model is not very different. For $g^{2} \sim \lambda \sim 0.1$ and $\alpha \simeq 0.25$ we have $M \simeq 2 \times 10^{-3} M_{P} \simeq 2 \times 10^{16} \mathrm{GeV}$, i.e., the grand unified theory (GUT) scale.

The spectrum (15) has a tilt given by

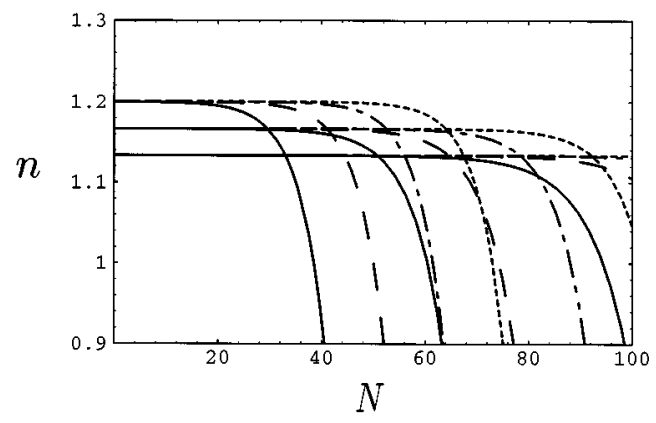

FIG. 1. The tilt of the spectrum (18) as a function of the number of $e$ folds to the end of inflation, for $N_{\text {hor }}=55$ and parameters $\alpha=0.3,0.25,0.2$, from top to bottom. The black, dashed, dotteddashed, and dotted lines for each $\alpha$ correspond to the ratio $g^{4} / \lambda=0.1,1.0,10,100$, respectively. Note that the tilt rises very quickly to its asymptotic value.

$$
n-1=\frac{d \ln \mathcal{P}_{\mathcal{R}}}{d \ln k}=\frac{2 \alpha}{3}\left(1-\frac{4 \pi \alpha \phi^{2}}{3 M_{P}^{2}}\right),
$$

which vanishes at

$$
\phi=\phi_{\min } \equiv \sqrt{\frac{3}{4 \pi \alpha}} M_{P},
$$

where the spectrum has a minimum. Later on, the tilt will acquire the asymptotic value $n_{\text {as }}=1+2 \alpha / 3$. This is reflected in Fig. 1, where it is seen how the tilt grows as a function of the number of $e$ folds to the end of inflation, for various values of $\alpha$. As we increase the ratio $g^{4} / \lambda$, the asymptotic tilt is more quickly approached.

Using the constraint (16) it is possible to find an expression for $N_{\max }$, as a function of $N_{\text {hor }}$ :

$$
N_{\text {max }} \simeq \frac{12}{\alpha} \ln 10-N_{\text {hor }}+\frac{3}{2 \alpha} \ln \left(\frac{27}{8 \pi \alpha^{4}} \frac{g^{4}}{\lambda}\right) .
$$

In Fig. 2 we have plotted the asymptotic value of the tilt $n_{\text {as }}=1+2 \alpha / 3$ as a function of $N_{\max }$, for various values of $g^{4} / \lambda$. As we increase this ratio, larger asymptotic tilts can be obtained for smaller values of $N_{\max }$. Thus strongly tilted hybrid models generically predict a small number of $e$ folds

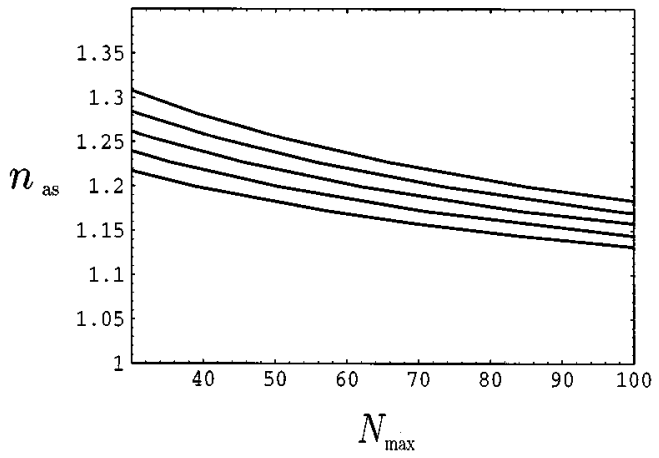

FIG. 2. The asymptotic tilt of the spectrum as a function of the maximum number of $e$ folds, $N_{\max }$, for $N_{\text {hor }}=55$. The five lines correspond to the ratio $g^{4} / \lambda=100,10,1,0.1,0.01$, from top to bottom. 
of inflation. This is another feature of the model that could be useful for open inflation. For example, depending on the parameters of the theory and the efficiency of reheating, the minimum of the spectrum of curvature perturbations (19) for $\alpha \sim 0.25$ could lie on scales comparable to the scale of our present horizon. If the minimum corresponds to the horizon scale, the spectrum on this scale will be practically flat. For $M \simeq 2 \times 10^{-3}, M_{P} \simeq 2 \times 10^{16} \mathrm{GeV}, \quad \alpha=0.25, \quad$ and $g^{2} \simeq \lambda \simeq 0.1$, we find $N_{\max } \sim 60$, which is of the order of the number of $e$ folds corresponding to the scale of the horizon. In this case, the scalar spectrum is nearly scale invariant, see Eq. (18), on those scales, but soon rises to an asymptotic value $n_{\mathrm{as}} \simeq 1.17$ on smaller scales. This choice of parameters [22] is not unique; e.g., one can have a larger $\alpha=0.3$, for $g=1$ and $\lambda=0.01$, which gives $M=6 \times 10^{15} \mathrm{GeV}$ and $n=1.2$ on all scales within the horizon, as you can see from Fig. 1. In this case, $N_{\max }=85$, which is much larger than $N_{\text {hor }}=55$.

\section{OPEN HYBRID INFLATION}

The new ingredient here is a period of false vacuum inflation prior to the stage of hybrid inflation. This could be accounted for by a symmetry breaking field $\sigma$ which is trapped in a false vacuum and eventually tunnels through to the true vacuum via the nucleation of a bubble. One of the possible models describing this process has the effective potential [3]

$$
V(\sigma, \phi)=\frac{M^{\prime 2} \sigma^{2}}{2}-\sqrt{\lambda^{\prime}} M^{\prime} \sigma^{3}+\frac{\lambda^{\prime} \sigma^{4}}{4}+\frac{h^{2} \phi^{2} \sigma^{2}}{2}+\widetilde{V},
$$

where we have included a coupling between $\sigma$ and $\phi$ to ensure the synchronization of the two fields after tunneling, in order to prevent large temperature fluctuations in the CMB [3]. The constant $\widetilde{V} \sim M^{\prime 4} / \lambda^{\prime}$ is added to make the effective potential vanish in its global minimum.

The combined effective potential for all fields in our model looks as

$$
\begin{aligned}
V(\sigma, \phi, \psi)= & -\frac{M^{2} \psi^{2}}{2}+\frac{\lambda \psi^{4}}{4}+\frac{M^{4}}{4 \lambda} \exp \left(\frac{4 \pi \alpha \phi^{2}}{3 M_{P}^{2}}\right)+\frac{M^{\prime 2} \sigma^{2}}{2} \\
& -\sqrt{\lambda^{\prime}} M^{\prime} \sigma^{3}+\frac{\lambda^{\prime} \sigma^{4}}{4}+\frac{\phi^{2}}{2}\left(g^{2} \psi^{2}+h^{2} \sigma^{2}\right)+\widetilde{V}
\end{aligned}
$$

Even though this model may look complicated, it is basically very simple: $V(\sigma, \phi, \psi)$ is the sum of the effective potential for the tilted hybrid inflation and the potential (21) for open inflation. The global minimum of this potential is at $\phi=0$, $\psi=M / \sqrt{\lambda}, \quad \sigma=\frac{1}{2}(3+\sqrt{5}) M^{\prime} / \sqrt{\lambda^{\prime}}$; the effective potential vanishes there. We suppose that $M / \sqrt{\lambda}, M^{\prime} / \sqrt{\lambda^{\prime}}, M / g$, $M^{\prime} / h \ll M_{P}$.

Now let us study the combined evolution of all three fields. Suppose that initially the scalar field $\phi$ is very large, $\phi \gg M / g, M^{\prime} / h$. At that time the minimum of the effective potential is at $\psi=\sigma=0$, and the fields roll there. The minimum at $\sigma \neq 0$ appears and becomes deeper than the minimum at $\sigma=0$ only for $\phi<\phi_{*} \equiv M^{\prime} / h$, and the phase transi- tion to $\psi \neq 0$ occurs at $\phi<\phi_{c} \equiv M / g$. Let us consider the model with $M^{\prime} / h>M / g$, i.e., $\phi_{*}>\phi_{c}$. In this case the first phase transition occurs when the field $\phi$ rolls down to $\phi_{*}$, and the bubbles of the field $\sigma$ containing open universes inside each of them are formed. Inside each bubble, the scalar field $\phi$ continues rolling down, driving the second stage of inflation which eventually ends when the field $\phi$ reaches $\phi_{c}$. If the tunneling event is very improbable, then other bubbles will not collide with ours in our past light cone and we could be living inside such a bubble.

The second stage of inflation inside each of the bubbles, which occurs when the field $\phi$ rolls down from $\phi_{c}$ to $\phi_{*}$, can be approximately described by the tilted hybrid inflation model described in the previous section. The number of $e$ folds of hybrid inflation inside the bubble is then given by

$$
N \approx \frac{3}{\alpha} \ln \frac{\phi_{*}}{\phi_{c}}=\frac{3}{\alpha} \ln \frac{g M^{\prime}}{h M} .
$$

For an open universe we require $N=55 \pm 5$.

However, one should take into account also an additional contribution of the term $h^{2} \phi^{2} \sigma^{2} / 2 \sim h^{2} \phi^{2} M^{\prime 2} / 2 \lambda^{\prime}$ to the effective mass of the scalar field $\phi$, as well as the contribution of the false vacuum energy density of the field $\sigma$ to the Hubble constant. Fortunately, under the following conditions these corrections are small and can be neglected:

$$
\begin{aligned}
\frac{h^{2} M^{\prime 2}}{\lambda^{\prime}} & \ll \frac{2 \pi \alpha M^{4}}{3 \lambda M_{P}^{2}}, \\
\frac{M^{\prime 4}}{\lambda^{\prime}} & \ll \frac{M^{4}}{\lambda} .
\end{aligned}
$$

Using Eqs. (16) and (23), the first condition can be written as

$$
\frac{h^{4}}{\lambda^{\prime}} \ll \frac{8 \pi \alpha^{3}}{9} 10^{-8} \text {. }
$$

All conditions will be satisfied if, for instance, one takes the values $\alpha=0.25, g^{2}=\lambda=0.1$, and $M=2 \times 10^{16} \mathrm{GeV}$, as in the previous section, and $\lambda^{\prime} \geqslant 0.1, h \sim 10^{-3}$, and $M^{\prime} \sim 10^{16} \mathrm{GeV}$. In such a case all the results of the previous section remain valid for our model. Thus we have found a working model of open universe that produces metric perturbations with a blue spectrum, as described in Sec. II.

\section{TEMPERATURE ANISOTROPIES}

Until recently, observations of the CMB anisotropies provided just a few constraints on the parameters of inflationary models, mainly from the amplitude and the tilt of both scalar and tensor perturbations' spectrum. Nowadays, with several experiments looking at different angular scales, we have much more information about both spectra as well as other cosmological parameters such as $\Omega_{0}, H_{0}, \Omega_{B}$, etc. In the near future, most of these parameters will be known with better than a few \% accuracy, thanks to the new generation of microwave anisotropy satellites, MAP [28] and COBRAS/ SAMBA [29].

Quantum fluctuations of the inflaton field $\phi$ during inflation produce long-wavelength scalar curvature perturbations and tensor (gravitational wave) perturbations, which leave 
their imprint in the CMB anisotropies. We will only consider here the scalar component and leave the tensor for a future publication. We will use $\mathcal{R}$ to denote the scalar metric perturbation on comoving hypersurfaces. Open inflation generates three different types of scalar modes: those that cross outside during the second stage of inflation and constitute a continuum of subcurvature modes [7,2], a discrete supercurvature mode [8], associated with the open de Sitter vacuum [10], and a mode associated with the bubble wall fluctuations at tunneling $[12,13,30]$, all of which induce temperature anisotropies in the microwave background.

In order to compare with observations we have to compute the effect that such a scalar metric perturbation has on the temperature anisotropies of the CMB (expanded in spherical harmonics):

$$
\frac{\delta T}{T}(\theta, \phi)=\sum_{l m} a_{l m} Y_{l m}(\theta, \phi) .
$$

In the context of an open universe, the main effect on large scales comes from the integrated Sachs-Wolfe effect [31]. The angular power spectrum $C_{l} \equiv\left\langle\left|a_{l m}\right|^{2}\right\rangle$ has contributions coming from the continuum of scalar subcurvature modes, the supercurvature mode, and the bubble wall mode:

$$
C_{l}=C_{l}^{(C)}+C_{l}^{(S)}+C_{l}^{(W)} .
$$

The contribution of each mode to the $C_{l}$ 's is measured by a window function $W_{q l}$, given by [31]

$$
5 W_{q l}=\Pi_{q l}\left(\eta_{0}\right)+6 \int_{0}^{\eta_{0}} d r F^{\prime}\left(\eta_{0}-r\right) \Pi_{q l}(r)
$$

for the subcurvature modes, where

$$
F(\eta)=5 \frac{\sinh ^{2} \eta-3 \eta \sinh \eta+4(\cosh \eta-1)}{(\cosh \eta-1)^{3}},
$$

and $\eta_{0}=\cosh ^{-1}\left(2 / \Omega_{0}-1\right)$ is the distance to the last scattering surface. Here $\Pi_{q l}(r)$ are the radial eigenfunctions of the Laplacian, with eigenvalue $-k^{2}=-\left(1+q^{2}\right)$, see Ref. [32]. The curvature scale corresponds to $k=1$. For subcurvature modes, $q^{2}>0$, while for supercurvature modes, $q^{2}<0$. There is an analogous expression to Eq. (28) for the supercurvature modes' window functions $\bar{W}_{|q| l}$ in terms of the eigenfunctions $\bar{\Pi}_{|q| l}(r)$. The corresponding expressions can be found in the appendix of Ref. [16], where a similar analysis was performed for the induced gravity open inflation model.

\section{A. Subcurvature modes}

Those fluctuations that cross the Hubble scale during the second stage of inflation give rise to a continuum spectrum of subcurvature scalar metric perturbations $[1,10]$ which can be computed in the case of hybrid inflation:

$$
\begin{gathered}
\mathcal{P}_{\mathcal{R}}=A_{C}^{2} \operatorname{coth}(\pi q), \\
A_{C}^{2}=\frac{3 g^{2} M^{2} e^{-2 \alpha N / 3}}{2 \pi \lambda \alpha^{2} M_{P}^{2}} .
\end{gathered}
$$

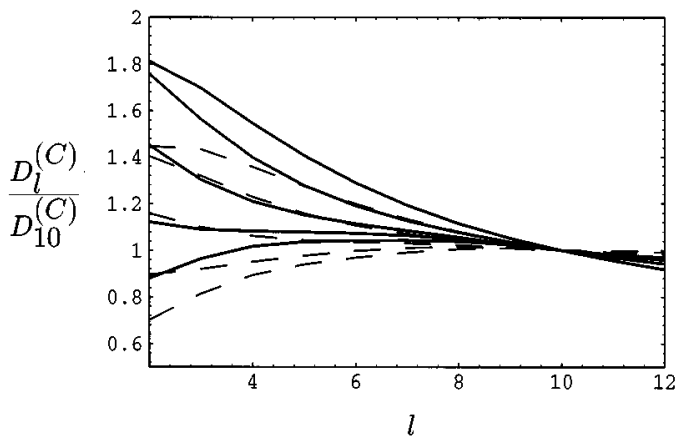

FIG. 3. The first 12 multipoles of the angular power spectrum associated with the continuum modes $D_{l}^{(C)}$ normalized to the tenth multipole, for $\Omega_{0}=0.2$ to 0.6 from top to bottom at low $l$. The thick lines correspond to a scale invariant $n=1$ spectrum, while the thin dashed lines correspond to a tilted $n=1.15$ spectrum.

The extra coth factor is due to the spatial curvature immediately after tunneling $[7,10]$. As inflation progresses inside the bubble, and the corresponding scales acquire values $q \gg 1$, the spectrum recovers the usual power-law behavior of flat inflation. The spectral tilt can then be evaluated as in Eq. (18), $n=1+2 \alpha / 3$. Note that the tilt is always greater than 1 in this model.

The angular power spectrum for the continuum modes can be written as [7]

$$
C_{l}^{(C)}=2 \pi^{2} \int_{0}^{\infty} \frac{d q}{q\left(1+q^{2}\right)} \mathcal{P}_{\mathcal{R}}(q) W_{q l}^{2}
$$

where $\mathcal{P}_{\mathcal{R}}(q)$ is the primordial curvature perturbations' spectrum (30) and the window function $W_{q l}$ can be computed from Eq. (28) with eigenfunctions given in Ref. [32,16].

We can compute the large angle power spectrum for different values of $\Omega_{0}$ in the low density range $0.2 \leqslant \Omega_{0} \leqslant 0.6$ for a scale invariant $n=1$ spectrum, as well as for a tilted $n=1.15$ spectrum. In Fig. 3 we show the first twelve multipoles, adopting the notation $D_{l}=l(l+1) C_{l}$. Note that the quadrupoles of the two spectra actually differ by a factor as large as 1.25 , for any value of $\Omega_{0}$. Clearly, a tilted $n>1$ density perturbation spectrum produces a flatter power spectrum in an open universe. We have computed the large scale power spectrum including only the Sachs-Wolfe effect, as appropriate to the lowest multipoles, while ignoring the effect of oscillations in the photon-baryon fluid, which is known to induce and effective extra tilt of about 0.15 , as it rises to the first acoustic peak at [33]

$$
l_{\text {peak }} \simeq 220 \Omega_{0}^{-1 / 2} .
$$

A future precise determination of $l_{\text {peak }}$ will allow us to conclude whether the universe is indeed open [34].

In order to compare our predictions with observations we have to know the amplitude and shape of the power spectrum of temperature fluctuations in the microwave background. The normalization to COBE [35] for tilted open models has recently been given by Bunn and White [36], under the assumption that only the continuum modes are important. They specify a quantity $\delta_{H}$, which measures the normalization of 
the present matter power spectrum in an open universe. The preferred value depends on $n$ and $\Omega_{0}$ [36]:

$$
\begin{aligned}
& \delta_{H}\left(n, \Omega_{0}\right)=1.95 \times 10^{-5} \Omega_{0}^{-0.35-0.19 \ln \Omega_{0}-0.17(n-1)} \\
& \times \exp \left[-(n-1)-0.14(n-1)^{2}\right] .
\end{aligned}
$$

We will consider values of the tilt away from 1, as well as low density universes, so both dependencies in principle need to be taken into account. However, the dependencies are not that strong for the values we are considering, so it is enough to take $\delta_{H} \simeq 2 \times 10^{-5}$, in order to determine the model's parameters. In the future, a precise knowledge of both $\Omega_{0}$ and $n$ will allow a better determination of the parameters. In an open universe $\delta_{H}$ is related to $\mathcal{P}_{\mathcal{R}}$ as [36]

$$
\delta_{H}=\frac{2}{5} \mathcal{P}_{\mathcal{R}}^{1 / 2} \frac{g\left(\Omega_{0}\right)}{\Omega_{0}},
$$

where $g\left(\Omega_{0}\right)$ is a function measuring the suppression in the growth of perturbations relative to a critical-density universe

$$
\frac{g(\Omega)}{\Omega}=\frac{5}{2}\left[\frac{1}{70}+\frac{209 \Omega}{140}-\frac{\Omega^{2}}{140}+\Omega^{4 / 7}\right]^{-1},
$$

which tends to 1 as $\Omega_{0} \rightarrow 1$, and $\mathcal{P}_{\mathcal{R}}$ is evaluated at around the tenth multipole, where cosmic variance is small and $\operatorname{coth} \pi q \simeq 1$. The $\Omega_{0}$ dependence can give a factor of up to 1.5 in the region of interest, but we can ignore it as we do not require such accuracy. Reproducing the amplitude of temperature anisotropies gives the most important constraint on the parameters of the model,

$$
\frac{3 g M e^{-\alpha N / 3}}{2 \alpha \sqrt{\lambda} M_{P}} \simeq 10^{-4}
$$

For $N \simeq 55$ on the scale of the horizon, and very natural values of the couplings, $g^{2} \sim \lambda \sim 0.1$, we have

$$
M \simeq 2 \times 10^{-3} M_{P} \simeq 2 \times 10^{16} \mathrm{GeV},
$$

the GUT scale. If the scalar field $\phi$ has a bare mass $m$, it should be smaller than

$$
\sqrt{\alpha} H_{0} \simeq 10^{-5} M_{P} .
$$

As long as $m$ is smaller than $\sqrt{\alpha} H_{0}$, nothing depends on this parameter, so we do not need to fine tune $m$ in order to obtain adiabatic scalar perturbations with a blue spectrum. Since $H_{0} \ll M$, hybrid inflation will end with a sudden transition to the symmetry-breaking phase of the triggering field, without a second stage of inflation such as the one discussed in Refs. [24,25].

\section{B. Supercurvature mode}

Apart from the continuum of subcurvature modes, in open inflation we also have a contribution to the microwave background anisotropies coming from a discrete supercurvature mode,

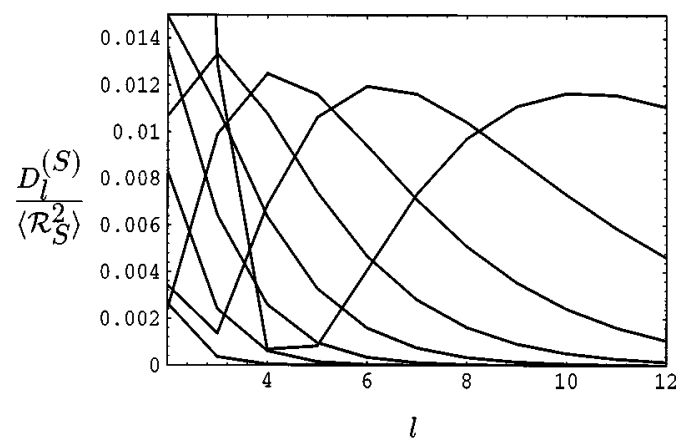

FIG. 4. The first 12 multipoles of the angular power spectrum associated with the supercurvature modes $D_{l}^{(S)}$ normalized to the curvature perturbation amplitude $\left\langle\mathcal{R}_{S}^{2}\right\rangle$ for $\Omega_{0}=0.2$ to 0.9 from top right to bottom left. The quadrupole does not necessarily dominate the power spectrum for small $\Omega_{0}$.

$$
k^{2}=1-\left[\left(\frac{9}{4}-\frac{m_{F}^{2}}{H_{F}^{2}}\right)^{1 / 2}-\frac{1}{2}\right]^{2},
$$

which appears in the spectrum of the inflaton field in open de Sitter when $m_{F}^{2}<2 H_{F}^{2}$ in the false vacuum [7]. The metric perturbation for this mode is given by [14]

$$
\left\langle\mathcal{R}_{S}^{2}\right\rangle \simeq A_{C}^{2} \frac{H_{F}^{2}}{H_{T}^{2}} .
$$

The angular power spectrum of temperature anisotropies induced by this supercurvature mode can be written as

$$
D_{l}^{(S)}=l(l+1) C_{l}^{(S)}=4 \pi\left\langle\mathcal{R}_{S}^{2}\right\rangle \bar{W}_{1 l}^{2},
$$

where $\bar{W}_{1 l}$ is the window function for this $|q|=1$ mode, see Ref. [16], where the correctly normalized eigenfunctions were given.

We have computed the angular power spectrum $D_{l}^{(S)}$ for different values of $\Omega_{0}$ in the range $0.2 \leqslant \Omega_{0} \leqslant 0.9$, as shown in Fig. 4 for the first twelve multipoles. We have found $D_{l}^{(S)} /\left\langle\mathcal{R}_{S}^{2}\right\rangle$ to be two orders of magnitude smaller than the continuum spectrum, see Fig. 6. Since we want the supercurvature mode contribution to the power spectrum to be smaller than that of the continuum modes, we need

$$
\left\langle\mathcal{R}_{S}^{2}\right\rangle<100 A_{C}^{2}
$$

This imposes only a very mild constraint on the parameters of the model,

$$
\frac{H_{F}^{2}}{H_{T}^{2}}<100,
$$

which is easy to satisfy for reasonable values of the parameters. Since we are considering that the tunneling occurs at $\phi \simeq \phi_{*}$, where the two minima of the tunneling potential are degenerate, we have $H_{F} \simeq H_{T}$ and therefore the bound is easily satisfied. 


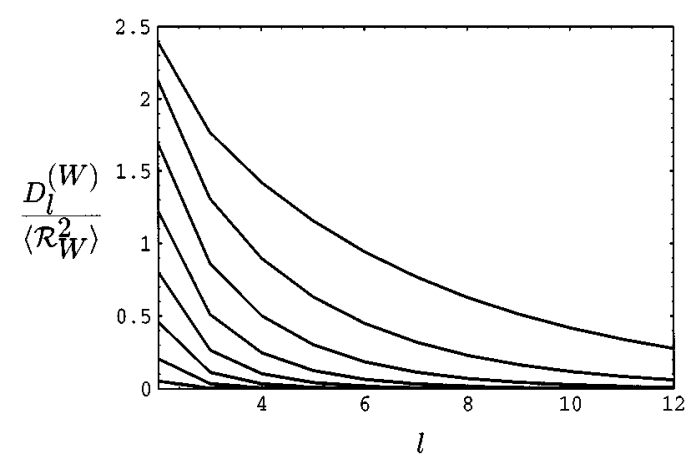

FIG. 5. The first 12 multipoles of the angular power spectrum associated with the bubble wall modes $D_{l}^{(W)}$ normalized to the curvature perturbation amplitude $\left\langle\mathcal{R}_{W}^{2}\right\rangle$, for $\Omega_{0}=0.2$ to 0.9 from top to bottom. The quadrupole dominates the $C_{l}^{(W)}$ power spectrum for all $\Omega_{0}$.

\section{Bubble wall mode}

Apart from the continuum of subcurvature modes and the discrete supercurvature mode, we expect also a contribution from the bubble wall fluctuations $[12,13,30]$. These fluctuations contribute as a transverse traceless curvature perturbation $k^{2}=-3$ mode, which nevertheless behaves as a homogeneous random field, see Refs. [11,37].

The curvature perturbation amplitude for this bubble wall mode can be computed from $[12,10]$

$$
\left\langle\mathcal{R}_{W}^{2}\right\rangle=\frac{3 \kappa^{2} H_{T}^{2}}{16 \pi^{2} \alpha^{2} \beta}\left[\alpha^{2}+\left(1+\alpha^{2} \beta\right)^{2}\right]^{1 / 2},
$$

where $\kappa^{2}=8 \pi G$ and

$$
\alpha=\frac{\kappa^{2} S_{1} H_{T}}{H_{F}^{2}-H_{T}^{2}}, \quad \beta=\frac{H_{F}^{2}-H_{T}^{2}}{4 H_{T}^{2}} .
$$

Here $S_{1}$ is the contribution to the bounce action coming from the bubble wall. At tunneling $\phi=\phi_{*}$, the potential (21) becomes

$$
U(\sigma)=\frac{\lambda^{\prime}}{4} \sigma^{2}\left(\sigma-\sigma_{0}\right)^{2},
$$

where $\sigma_{0}=2 M^{\prime} / \sqrt{\lambda^{\prime}}$. Thus $S_{1}$ can be computed as [12]

$$
S_{1}=\int_{0}^{\sigma_{0}} d \sigma\left\{2\left[U(\sigma)-U_{F}\right]\right\}^{1 / 2}=\frac{2 \sqrt{2} M^{\prime 3}}{3 \lambda^{\prime}} .
$$

Note that in the limit $\alpha^{2} \beta \ll 1$ of small gravitational effects at tunneling, we recover the result of Ref. [3]:

$$
\left\langle\mathcal{R}_{W}^{2}\right\rangle=\frac{3 H_{T}^{2}\left(H_{F}^{2}-H_{T}^{2}\right)}{4 \pi^{2} \kappa^{2} S_{1}^{2}} .
$$

However, in the opposite limit of strong gravitational effects $\alpha^{2} \beta \gg 1$, we have [12]

$$
\left\langle\mathcal{R}_{W}^{2}\right\rangle=\frac{3 \kappa^{2} H_{T}^{2}}{16 \pi^{2}}
$$

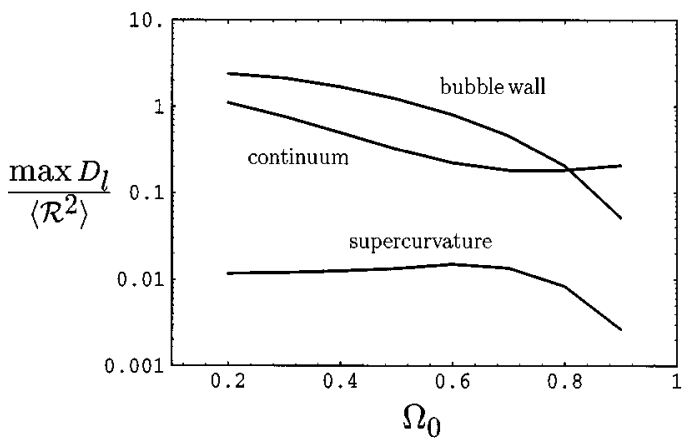

FIG. 6. The largest multipole of the angular power spectrum, normalized to the corresponding metric perturbation, for the continuum, supercurvature, and bubble wall modes, as a function of $\Omega_{0}$. While the continuum modes do not change much as we approach $\Omega_{0}=1$, the supercurvature and bubble wall modes decrease exponentially.

The contribution of the bubble wall mode to the angular power spectrum can be written as

$$
D_{l}^{(W)}=l(l+1) C_{l}^{(W)}=\frac{4 \pi\left\langle\mathcal{R}_{W}^{2}\right\rangle}{(l+2)(l-1)} \bar{W}_{2 l}^{2},
$$

where $\bar{W}_{2 l}$ is the window function for this $|q|=2$ mode, see Ref. [16] for the correctly normalized eigenfunctions.

We have computed the angular power spectrum $C_{l}^{(W)}$ for different values of $\Omega_{0}$ in the range $0.2 \leqslant \Omega_{0} \leqslant 0.9$, as shown in Fig. 5 for the first twelve multipoles. We have found $l(l+1) C_{l}^{(W)} /\left\langle\mathcal{R}_{W}^{2}\right\rangle$ to be of the same order of magnitude or smaller than the continuum spectrum, see Fig. 6. Since we want the bubble wall mode contribution to the power spectrum to be smaller than that of the continuum modes, we need

$$
\left\langle\mathcal{R}_{W}^{2}\right\rangle<A_{C}^{2}
$$

This imposes only a very mild constraint on the parameters of the model. For $\alpha^{2} \beta \ll 1$, we would have

$$
\alpha e^{\alpha N / 3}<\frac{3 g S_{1}}{M\left(U_{F}-U_{T}\right)^{1 / 2}},
$$

which is easy to satisfy for sufficiently large $M^{\prime}$, see Ref. [3]. However, since we are considering the tunneling when $H_{F} \simeq H_{T}$, we have $\alpha^{2} \beta \gg 1$ and the amplitude of the metric perturbation (50) becomes

$$
\left\langle\mathcal{R}_{W}^{2}\right\rangle=\frac{1}{\lambda} \frac{M^{4}}{M_{P}^{4}},
$$

which could indeed be smaller than $A_{C}^{2}$ for the parameters of tilted hybrid models $\lambda \simeq 0.1$ and $M \simeq 2 \times 10^{-3} M_{P}$ discussed in Sec. II.

\section{CONCLUSIONS}

In this paper we have studied an open inflation model in which the second period of expansion inside the bubble is driven by tilted hybrid inflation [22]. This model has a char- 
acteristic signature of a tilted $n>1$ spectrum. Such a spectrum is nowadays the best candidate for explaining the CMB anisotropies in case the universe is wide open, with $\Omega_{0}<0.4$ [15].

We have computed the angular power spectrum of temperature fluctuations in the CMB induced by the continuum of scalar subcurvature modes, as well as the de Sitter supercurvature mode and the bubble wall mode. We have found a set of constraints that the parameters of such a model should satisfy in order to agree with observations. The parameters of our model satisfy all the constraints in a natural way. The contribution of a primordial spectrum of gravitational waves to the complete angular power spectrum of temperature anisotropies and the associated constraints on the parameters of the open hybrid model will be discussed in a separate publication.

Note that our model requires the existence of three scalar fields, which is a long shot from the simplest inflationary models describing only one scalar field. On the other hand, our model represents a simple combination of the hybrid inflation scenario and the theory of a scalar field $\sigma$ with a metastable vacuum state $\sigma=0$. The hybrid inflation scenario recently became very popular. It allows inflation to occur at sub-Planckian values of scalar fields, which makes it easier to realize it in the context of supersymmetric models. The scalar field $\sigma$ with a metastable vacuum state is necessary for open inflation anyway. Thus our model consists of only two building blocks, each of which is rather natural in the context of inflationary cosmology. There are many ways to combine these two models together, so the model described in this paper is not unique, and it is quite possible that a simpler and more elegant model can be proposed. Our main purpose here was simply to show that open inflation models with blue spectra do exist. On the other hand, the tilted hybrid inflation which we used in our scenario has a naturally short duration of inflation after the tunneling with the open universe formation. This is exactly what we need in order to have an inflationary universe with $\Omega<1$.

In principle, one can obtain a tilted spectrum of perturbations in an entirely different way, by combining the open inflation model with the recently proposed model of Ref. [26], in which one may have strongly tilted isocurvature or adiabatic non-Gaussian perturbations. It might be possible to obtain a model with all desirable properties that will require only two scalar fields.

To avoid misunderstandings, we should emphasize that we sincerely hope that at the end of the day observational data will show that the universe is flat, and that perturbations of the metric are scale invariant, as suggested by the simplest inflationary models. However, when the universe was built we were not consulted. Our final goal is to propose an internally consistent theory that will correctly describe observational data. We are very encouraged that a consistent theory of an open universe does exist. So far this theory has been formulated only in the context of inflationary cosmology. This means that the consistent cosmological models describing a large nearly homogeneous universe with $\Omega<1$ do not contradict inflation and, moreover, they have so far been constructed only within the context of inflation. In this paper we have shown that it is not difficult to modify the spectrum of perturbations of the metric in such models, which may be necessary to make them consistent with observations.

\section{ACKNOWLEDGMENTS}

The work of A.L. was supported by NSF Grant No. PHY9219345. This work was also supported by a NATO Collaborative Research Grant, Ref. CRG.950760.
[1] J. R. Gott, Nature (London) 295, 304 (1982); J. R. Gott III and T. S. Statler, Phys. Lett. 136B, 157 (1984).

[2] M. Bucher, A. S. Goldhaber and N. Turok, Phys. Rev. D 52, 3314 (1995); M. Bucher and N. Turok, ibid. 52, 5538 (1995); K. Yamamoto, M. Sasaki, and T. Tanaka, Astrophys. J. 455, 412 (1995).

[3] A. D. Linde, Phys. Lett. B 351, 99 (1995); A. D. Linde and A. Mezhlumian, Phys. Rev. D 52, 6789 (1995).

[4] W. L. Freedman et al., Nature (London) 371, 757 (1994); N. R. Tanvir et al., ibid. 377, 27 (1995).

[5] M. Bolte and C. J. Hogan, Nature (London) 376, 399 (1995).

[6] A. D. Linde, Sov. Phys. JETP 60, 211 (1984); Lett. Nuovo Cimento 39, 401 (1984); Ya. B. Zeldovich and A. A. Starobinsky, Sov. Astron. Lett. 10, 135 (1984); V. A. Rubakov, Phys. Lett. 148B, 280 (1984); A. Vilenkin, Phys. Rev. D 30, 549 (1984).

[7] M. Sasaki, T. Tanaka, and K. Yamamoto, Phys. Rev. D 51, 2979 (1995).

[8] D. H. Lyth and A. Woszczyna, Phys. Rev. D 52, 3338 (1995).

[9] J. García-Bellido, A. R. Liddle, D. H. Lyth, and D. Wands, Phys. Rev. D 52, 6750 (1995).

[10] K. Yamamoto, M. Sasaki, and T. Tanaka, Phys. Rev. D 54, 5031 (1996).
[11] T. Hamazaki, M. Sasaki, T. Tanaka, and K. Yamamoto, Phys. Rev. D 53, 2045 (1996).

[12] J. García-Bellido, Phys. Rev. D 54, 2473 (1996).

[13] J. Garriga, Phys. Rev. D 54, 4764 (1996).

[14] M. Sasaki and T. Tanaka, Phys. Rev. D 54, R4705 (1996).

[15] M. White and J. Silk, Phys. Rev. Lett. 77, 4704 (1996).

[16] J. García-Bellido and A. R. Liddle, Phys. Rev. D 55, 4603 (1997).

[17] A. D. Linde, Phys. Lett. B 259, 38 (1991); Phys. Rev. D 49, 748 (1994).

[18] A. R. Liddle and D. H. Lyth, Phys. Rep. 231, 1 (1993).

[19] E. J. Copeland, A. R. Liddle, D. H. Lyth, E. D. Stewart, and D. Wands, Phys. Rev. D 49, 6410 (1994).

[20] J. García-Bellido and D. Wands, Phys. Rev. D 54, 7181 (1996).

[21] For a comprehensive review, see D. H. Lyth, Report No. hep-ph/9609431, 1996 (unpublished).

[22] J. García-Bellido and A. Linde, Report No. CERN-TH-96-358, astro-ph/9612141, 1996 (unpublished).

[23] T. Tanaka and M. Sasaki, Osaka Report No. OU-TAP 48 astro-ph/9701053, 1997 (unpublished); M. Bucher and J. D. Cohn, this issue, Phys. Rev. D 55, 7461 (1997). 
[24] L. Randall, M. Soljačiıc, and A. H. Guth, Nucl. Phys. B472, 377 (1996).

[25] J. García-Bellido, A. D. Linde, and D. Wands, Phys. Rev. D 54, 6040 (1996).

[26] A. Linde and V. Mukhanov, Report No. SU-ITP-96-49, astro-ph/9610219, 1996 (unpublished).

[27] M. Dine, W. Fischler, and D. Nemeschansky, Phys. Lett. 136B, 169 (1984); G. D. Coughlan, R. Holman, P. Ramond, and G. G. Ross, ibid., 140B, 44 (1984); A. S. Goncharov, A. D. Linde, and M. I. Vysotsky, ibid. 147B, 279 (1984); M. Dine, L. Randall, and S. Thomas, Nucl. Phys. B458, 291 (1996).

[28] MAP Home Page at URL http://map.gsfc.nasa.gov/

[29] COBRAS/SAMBA Home Page at URL
http://astro.estec.esa.nl/SA-general/Projects/Cobras/ cobras.html

[30] J. D. Cohn, Phys. Rev. D 54, 7215 (1996).

[31] R. K. Sachs and A. M. Wolfe, Astrophys. J. 147, 73 (1967).

[32] E. R. Harrison, Rev. Mod. Phys. 39, 862 (1967); M. L. Wilson, Astrophys. J. 273, 2 (1983); L. F. Abbott and R. K. Schaefer, ibid. 308, 546 (1986).

[33] W. Hu and N. Sugiyama, Phys. Rev. D 51, 2599 (1995).

[34] G. Jungman, M. Kamionkowski, A. Kosowsky, and D. N. Spergel, Phys. Rev. D 54, 1332 (1996).

[35] C. L. Bennett et al., Astrophys. J. 464, L1 (1996).

[36] E. F. Bunn, A. R. Liddle, and M. White, Phys. Rev. D 54, 5917 (1996).

[37] J. García-Bellido, A. R. Liddle, D. H. Lyth, and D. Wands, Phys. Rev. D 55, 4596 (1997). 